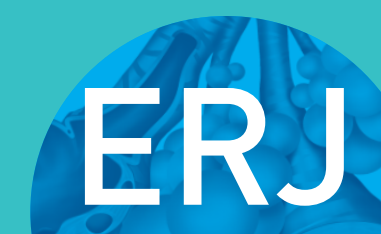

open research

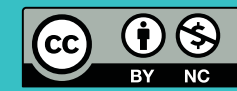

\section{Functional characterisation of bone marrow-derived mesenchymal stromal cells from COPD patients}

\author{
Winifred Broekman ${ }^{1}$, Helene Roelofs ${ }^{2}$, Maria C. Zarcone ${ }^{1}$, Christian Taube $^{1}$, \\ Jan Stolk ${ }^{1}$ and Pieter S. Hiemstra ${ }^{1}$
}

Affiliations: ${ }^{1}$ Dept of Pulmonology, Leiden University Medical Center, Leiden, The Netherlands. ${ }^{2}$ Dept of Immunohematology and Blood Transfusion, Leiden University Medical Center, Leiden, The Netherlands.

Correspondence: Winifred Broekman, Dept of Pulmonology, Leiden University Medical Center, Albinusdreef 2 , 2333 ZA Leiden, The Netherlands. E-mail: w.broekmanalumc.nl

ABSTRACT Autologous bone marrow-derived mesenchymal stromal cells (BM-MSCs) are evaluated for clinical use in chronic obstructive pulmonary disease (COPD) patients, but it is unclear whether COPD affects BM-MSCs.

To investigate this, BM-MSCs from nine COPD patients and nine non-COPD age-matched controls were compared with regard to immunophenotype, growth and differentiation potential, and migration capacity. Other functional assays included the response to pro-inflammatory stimuli and inducers of the nuclear factor (erythroid derived 2)-like 2 antioxidant response element (Nrf2-ARE) pathway, and effects on NCIH292 airway epithelial cells.

No significant differences were observed in terms of morphology, proliferation and migration, except for increased adipocyte differentiation potential in the COPD group. Both groups were comparable regarding mRNA expression of growth factors and inflammatory mediators, and in their potential to induce mRNA expression of epidermal growth factor receptor ligands in NCI-H292 airway epithelial cells. MSCs from COPD patients secreted more interleukin-6 in response to pro-inflammatory stimuli. Activation of the Nrf2ARE pathway resulted in a comparable induction of mRNA expression of four target genes, but the expression of the $\mathrm{NAD}(\mathrm{P}) \mathrm{H}$ :quinone oxidoreductase 1 gene NQO1 was lower in MSCs from COPD patients.

The observation that MSCs from COPD patients are phenotypically and functionally comparable to those from non-COPD controls implies that autologous MSCs can be considered for use in the setting of clinical trials as a treatment for COPD.

@ERSpublications

Phenotype and functional analysis of BM-MSCs from COPD patients supports their use in autologous MSC treatment http://ow.ly/TRtX3008t6c

This article has supplementary material available from openres.ersjournals.com

Received: July 162015 | Accepted after revision: May 062016

Support statement: This study was supported in part by a grant from the Lung Foundation Netherlands (grant number 6.1.14.009). Funding information for this article has been deposited with the Open Funder Registry.

Conflict of interest: Disclosures can be found alongside this article at openres.ersjournals.com

Copyright $\odot$ ERS 2016. This article is open access and distributed under the terms of the Creative Commons Attribution Non-Commercial Licence 4.0. 


\section{Introduction}

Mesenchymal stromal cells (MCSs) are defined as plastic adherent cells with the capacity to self-renew and differentiate into multiple lineages of the mesenchyme [1]. Importantly, it has been shown that MSCs stimulate recovery of damaged tissue via paracrine effects, potentiation of cell growth and wound healing, suppression of apoptosis, and possibly induction of endogenous progenitor cell potential [2]. In addition to their regenerative potential, MSCs have pleiotropic effects on several immune cells and can contribute to immune responses by modifying the inflammatory environment [3].

In light of these regenerative and immunomodulatory properties of MSCs, interest has arisen in the clinical application of MSCs as a treatment for chronic obstructive pulmonary disease (COPD). COPD is a heterogeneous disease, defined by persistent airflow limitation that is usually progressive. Mechanisms that contribute to COPD development include exaggerated inflammatory responses to inhaled noxious gases, imbalance between proteinases and proteinase inhibitors, and excessive oxidative stress [4]. On a cellular level, an imbalance of cell death and replenishment of structural cells ultimately results in tissue damage.

In COPD, MSC-based treatments have already been used in the context of clinical trials investigating both allogeneic [5] and autologous MSCs [6]. A concern when using MSCs is that donor-related factors might affect the therapeutic potential of MSCs. This is conceivable in MSCs from COPD patients, since COPD is considered to have a systemic component [7]. Indeed, in a preliminary report by JAHN et al. [8], functional differences between MSCs from COPD and control patients were observed. Alternatively, it has been hypothesised that altered MSC function contributes to the development of COPD [9].

Since autologous MSCs are currently used in clinical trials to evaluate their ability to induce (favourable) responses in lung tissue, it is relevant to know whether MSCs from COPD patients have similar properties and potential as MSCs from healthy donors. In this study this was investigated using in vitro cultures of bone marrow-derived MSCs (BM-MSCs) from COPD patients and age-matched non-COPD controls. We compared the MSC (immuno)phenotype and several functional parameters, including differentiation and migration, response to pro-inflammatory stimuli and inducers of the nuclear factor (erythroid derived 2)-like 2 antioxidant response element (Nrf2-ARE) pathway, and regenerative effects on airway epithelial cells.

\section{Materials and methods}

A more detailed description of the methods used for this study is provided in the supplementary material.

\section{Patients and ethical considerations}

BM-MSCs were obtained from COPD patients participating in a clinical trial to evaluate BM-MSCs for severe emphysema (ClinicalTrials.gov: NCT01306513) and from non-COPD controls [6].

Groups were matched for age (mean \pm SD: $53.1 \pm 6.4$ years for COPD versus $48.8 \pm 4.5$ years for non-COPD, $\mathrm{p}=0.12$ ) and sex (both groups three males out of nine). The control group included one Asian donor, all others were Caucasian. COPD donors had forced expiratory volume in $1 \mathrm{~s}<40 \%$ predicted, emphysema on computed tomography scan and had quit smoking at least 6 months prior to bone marrow aspiration. Controls had no known pulmonary disease and were never-smokers (the smoking history of three controls could not be traced). The use of MSCs for pre-clinical research was approved by the Medical Ethical Committee of the Leiden University Medical Center and donors consented to the possible use of their MSCs for this purpose.

\section{MSC cultures and characterisation}

MSC isolation from bone marrow and expansion in vitro was done following a previously published protocol [10], monitoring expansion rates during initial cultures. MSC immunophenotype was confirmed by fluorescence-activated cell sorter (FACS) analysis, differentiation potential was quantified by measuring adipocyte, osteoblast and calcium staining intensity of differentiated MSCs, and migration was assessed using electric cell-substrate impedance sensing (ECIS).

MSCs at passage 3 or 4 were used for experiments, after overnight incubation in serum-free medium.

\section{Stimuli}

Cigarette smoke extract (CSE) and sulforaphane (SFN) were used to induce the Nrf2-ARE pathway [11-13]. Tumour necrosis factor (TNF)- $\alpha$ and interleukin (IL)-1 $\beta$ (Peprotech, Rocky Hill, NJ, USA) at $20 \mathrm{ng} \cdot \mathrm{mL}^{-1}$ were used as pro-inflammatory stimuli to evaluate immune modulatory properties and growth factor induction. MSC conditioned medium (MSC-CM) was generated by culturing MSCs during $24 \mathrm{~h}$ in serum-free medium or in serum-free medium supplemented with TNF- $\alpha /$ IL- $1 \beta$ and was used to stimulate NCI-H292 airway epithelial cells. 
Quantitative real-time PCR and ELISA

MSCs were stimulated during $6 \mathrm{~h}$ for quantitative real-time PCR analysis or during $24 \mathrm{~h}$ for ELISAs. NCI-H292 airway epithelial cells were incubated during $9 \mathrm{~h}$ with MSC-CM to assess induction of mRNA expression of epidermal growth factor receptor (EGFR) ligands.

Following RNA extraction and cDNA synthesis, quantitative real-time PCR was performed in triplicate using primers for target genes (supplementary table E1) and relative gene expression compared with reference genes was calculated according to the standard curve method, with housekeeping genes selected based on geNorm software [14], i.e. $\beta_{2}$-microglobulin (B2M) and ribosomal protein S29 (RPS29) for MSCs and ATP synthase subunit $\beta$ (ATP5B) and ribosomal protein L13A (RPL13A) for NCI-H292 airway epithelial cells.

IL-6 (Sanquin, Amsterdam, The Netherlands) and IL-8 (R\&D Systems, Minneapolis, MN, USA) secretion was measured using ELISA, following the manufacturer's protocol. Optical density values were measured with a microplate reader.

\section{Statistical analysis}

Data analysis and composition of the figures were done using Prism 6.0 (GraphPad, La Jolla, CA, USA) and SPSS 20 (IBM, Armonk, NY, USA). Statistical analysis was performed using the Mann-Whitney U-test for analysis of differences between subject groups or the Wilcoxon matched-pairs signed-rank test for paired observations to compare conditions within groups. Differences were considered statistically significant at $\mathrm{p}<0.05$.

\section{Results}

BM-MSCs from COPD patients have the same (immunolphenotype as non-COPD controls, but have a stronger potential towards adipocyte differentiation

BM-MSCs from COPD patients and non-COPD controls were morphologically similar, displaying the characteristic spindle-shaped appearance (figure 1a). No differences were observed in immunophenotype (figure 1b), and all MSCs could be differentiated into adipocytes and osteoblasts, confirming their MSC phenotype. MSCs from COPD donors showed a significantly higher potential to differentiate into adipocytes than MSCs from non-COPD donors (figure 1c). No significant differences were observed in osteoblast differentiation and mineralisation potential. In six patients (four in the non-COPD group, two in the COPD group), no data were obtained for osteoblast differentiation, mineralisation or both, due to ruptures and subsequent detachment of the monolayer, caused by contractility of cells at the periphery of the well during differentiation.

Proliferation and migration potential of MSCs is not affected in MSCs from COPD patients Proliferation was assessed during expansion following initial isolation from bone marrow. No differences were observed between groups regarding the time between passages and the number of MSCs harvested per passage (figure $2 \mathrm{a}$ and $\mathrm{b}$ ). Migration was assessed as the potential of MSCs to cover the surface area of an electrode using ECIS analysis to measure resistance and capacitance. This showed that the migration potential of MSCs from COPD patients was similar to that of non-COPD controls (figure 2c).

\section{BM-MSC response to pro-inflammatory stimuli is similar between groups, except for higher IL-6} secretion in MSCs from COPD patients

Previously, MSCs were shown to express growth factors and immune mediators upon exposure to pro-inflammatory stimuli $[15,16]$. We therefore investigated whether this response was preserved in MSCs from COPD patients. In MSCs from COPD patients as well as non-COPD controls, incubation with TNF- $\alpha$ and IL- $1 \beta$ resulted in a significant induction of gene expression of amphiregulin (AREG), heparin-binding epidermal growth factor-like growth factor (HBEGF), fibroblast growth factor 2 (FGF2), chemokine (C-C motif) ligand 20 (CCL20), IL-6 (IL6), chemokine (C-X-C motif) ligand 8 (CXCL8) and TNF-stimulated gene 6 (TSG6) in both the COPD and non-COPD group (figure 3a). Furthermore, it increased transforming growth factor- $\alpha$ (TGFA) in the non-COPD group and hepatocyte growth factor $(H G F)$ in the COPD group (data not shown), and lowered expression of adrenomedullin (ADM1) in both groups (figure 3a). Between groups, no significant differences were observed. No significant induction was observed for other genes investigated (supplementary figure E1).

IL- 6 and IL- 8 protein secretion was measured in medium obtained from TNF- $\alpha$ - and IL-1 $\beta$-exposed MSCs. In unstimulated MSCs, IL-8 levels were below the detection limit and IL-6 secretion was comparable between groups (mean \pm SEM: $164 \pm 27.7 \mathrm{pg} \cdot \mathrm{mL}^{-1}$ for COPD versus $174 \pm 31.6 \mathrm{pg} \cdot \mathrm{mL}^{-1}$ for non-COPD, $\mathrm{p}=0.34$ ). Upon stimulation with pro-inflammatory cytokines, IL-8 and IL-6 secretion significantly increased in MSCs from both COPD and non-COPD donors. Differences between groups were observed, as MSCs 
a)

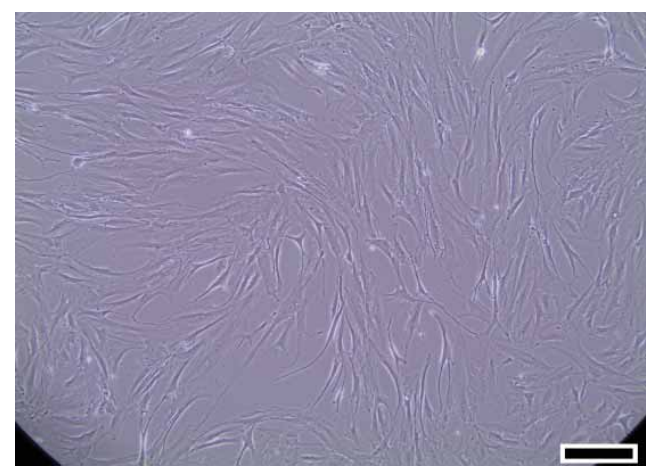

COPD

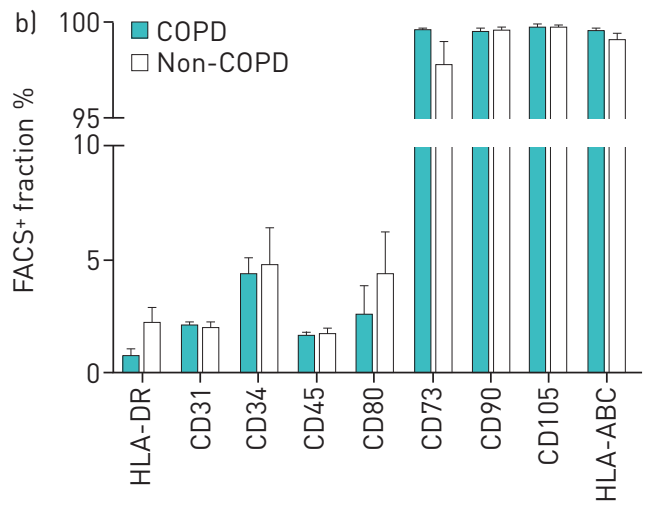

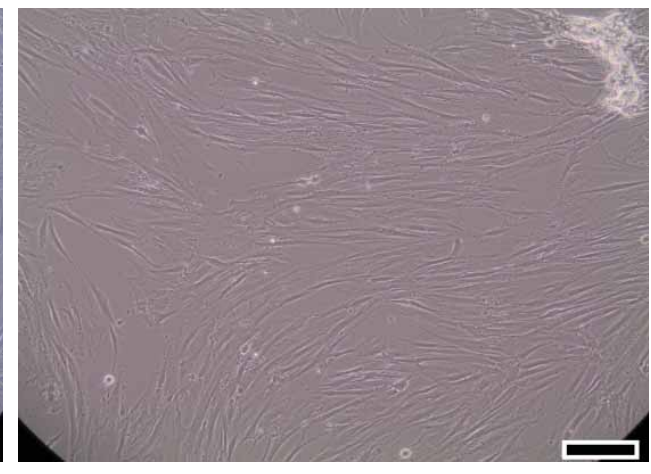

Non-COPD

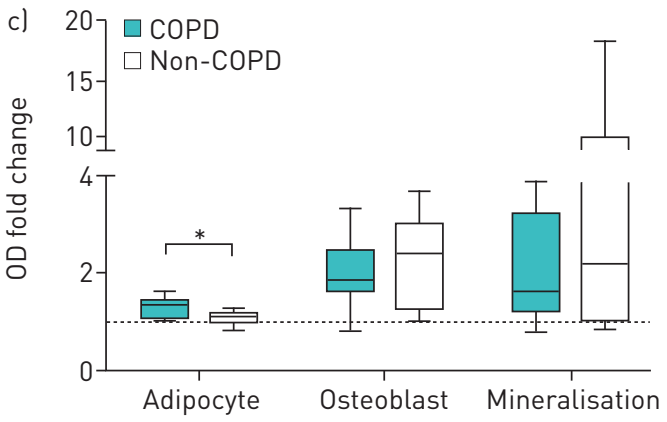

FIGURE 1 Mesenchymal stromal cell (MSC) phenotype from chronic obstructive pulmonary disease (COPD) and non-COPD patients. Bone marrow-derived MSCs (BM-MSCs) from COPD and non-COPD patients cultured in vitro were characterised by fluorescence-activated cell sorter (FACS) analysis and by their potential to differentiate into mesenchymal lineages. a) Morphology of MSCs from COPD and non-COPD donors: example of MSC culture. Scale bars $=100 \mu \mathrm{m}$. b) FACS data. Per antibody, data are shown as mean \pm SEM. $n=9$ per group. c) Differentiation into adipocytes and osteoblasts, and mineralisation potential. Staining intensity of Oil red 0 , alkaline phosphatase and Alizarin red (calcium) was quantified on a microplate reader, and per donor optical density (OD) values were calculated as fold change compared with control (co-cultured undifferentiated MSCs). Plots represent median, interquartile range, and minimum and maximum values. ${ }^{*}: p<0.05, n=9$ per group (except osteoblast/mineralisation: $\mathrm{n}=6-8$ per group).

from COPD patients secreted significantly higher amounts of IL- 6 and showed a trend towards higher IL-8 secretion $(\mathrm{p}=0.12)$ (figure $3 \mathrm{~b})$.

\section{Oxidative stress response in MSCs from COPD patients is comparable to that observed in MSCs from controls}

In COPD, insufficient antioxidant response has been implicated in disease pathogenesis [17]. Therefore, we evaluated the antioxidant response of MSCs from COPD patients, using CSE and SFN to activate the Nrf2-ARE pathway, and assessed induction of several downstream target genes involved in antioxidant response [11-13]. In both groups, incubation of MSCs with CSE resulted in a significant induction of haem oxygenase 1 (HMOX1), $\mathrm{NAD}(\mathrm{P}) \mathrm{H}$ :quinone oxidoreductase 1 (NQO1), and smoke and cancer-associated long noncoding RNA-1 (SCAL1). For HMOX1 and SCAL1, this induction was dose dependent. Glutathione peroxidase 2 (GPX2) expression decreased in CSE-exposed MSCs. Induced gene expression of NQO1 and SCAL1 was generally lower in MSCs from COPD patients, resulting in significant differences between groups in NQO1 expression at higher concentrations of CSE (figure 4a).

SFN significantly increased all analysed Nrf2-ARE-regulated target genes in non-COPD patients, whereas it only significantly induced HMOX1 and SCAL1 gene expression in MSCs from COPD patients (figure 4b). Despite this observation, groups did not significantly differ from each other in their response to SFN.

\section{Regenerative potential of MSCs from COPD patients is comparable to non-COPD controls}

MSC-CM from healthy donors induced EGFR ligand expression in NCI-H292 airway epithelial cells [15]. We assessed if MSC-CM from COPD patients has the same regenerative potential by investigating induction of mRNA expression of EGFR ligands in NCI-H292 airway epithelial cells, following incubation with MSC-CM ${ }^{\text {CTRL }}$ (from MSCs cultured in serum-free medium) or MSC-CM ${ }^{\text {STIM }}$ (from MSCs cultured in serum-free medium supplemented with TNF- $\alpha /$ IL-1 $\beta$ ). 

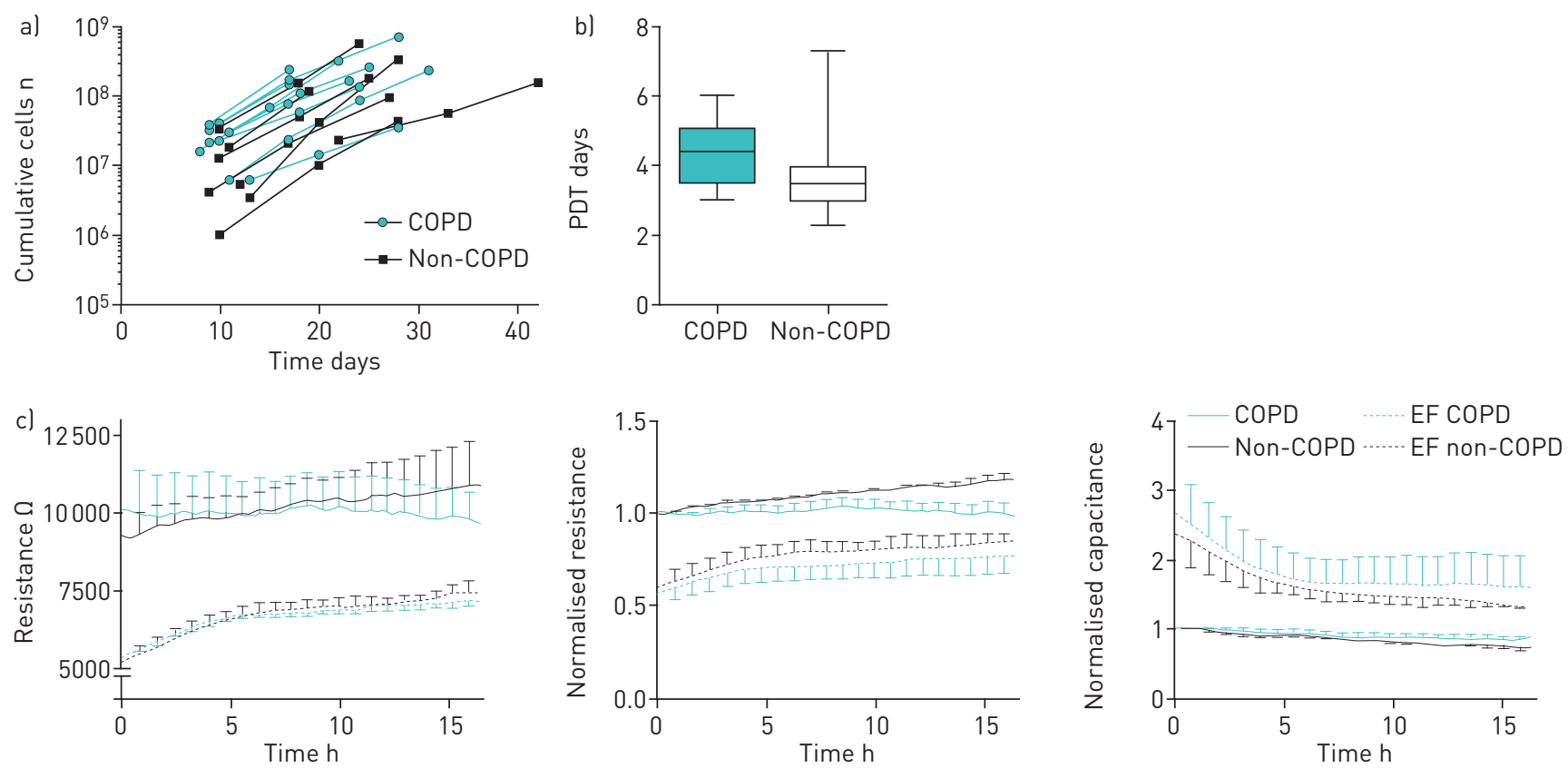

FIGURE 2 Mesenchymal stromal cell (MSC) proliferation and migration capacity. In vitro proliferation of MSCs from chronic obstructive pulmonary disease (COPD) and non-COPD patients was followed over time. a) Time needed for cultures to become near-confluent and the number of MSCs that were obtained per passage were assessed. b) Population doubling time (PDT) was calculated by dividing the natural logarithm of 2 by the exponent of growth. Plots represent median, interquartile range, and minimum and maximum values. $\mathrm{n}=9$ per group. c) Migration as assessed using electric cell-substrate impedance sensing (ECIS). MSCs were cultured in ECIS arrays in the presence of an electrical fence (EF), which prevented cell adherence across the electrode area. Resistance (at $500 \mathrm{~Hz}$ ) and capacitance (at $40 \mathrm{kHz}$ ) were measured continuously and followed up to $15 \mathrm{~h}$ after removal of the electrical fence ( $\mathrm{t}=0 \mathrm{~h}$ ). Restoration of the resistance and capacitance to control values (corresponding to full coverage of the electrode) was used as a measure for migration capacity of MSCs. Normalised data were obtained by correcting for the resistance/capacitance values obtained in control wells without an electric fence, at the time the electric fence was removed ( $t=0 \mathrm{~h}$ ). Data are presented as mean \pm SEM. $n=5-8$ per group.

Compared with control culture medium, both $\mathrm{MSC}^{-\mathrm{CM}^{\mathrm{CTRL}}}$ as well as MSC-CM ${ }^{\mathrm{STIM}}$ induced gene expression of the EGFR ligands AREG and HBEGF, and to a lesser extent of TGFA (data not shown), in NCI-H292 airway epithelial cells. In addition, both MSC-CM ${ }^{\text {CTRL }}$ and MSC-CM ${ }^{\text {STIM }}$ induced mRNA expression of the cell cycle regulator cyclin D1 (CCDN1), which is considered as a marker for cell proliferation. Compared with MSC-CM ${ }^{\mathrm{CTRL}}$, no significant differences were observed between the COPD and non-COPD groups in the potential of MSC-CM ${ }^{\text {STIM }}$ to induce EGFR ligands in NCI-H292 airway epithelial cells, although there were differences in the significance of effects of MSC-CM ${ }^{\text {STIM }}$ from non-COPD and COPD patients (figure 5).

\section{Discussion}

In this study we show that MSCs from patients with COPD are largely comparable in phenotype and function to MSCs from non-COPD controls. MSCs from COPD patients responded similarly to those from non-COPD controls for the majority of the investigated parameters, including (immuno)phenotype, proliferation and migration potential, response to pro-inflammatory stimuli, and effects on airway epithelial cells. However, we did observe differences in adipocyte differentiation, IL-6 secretion in response to pro-inflammatory stimuli and induction of gene expression of NQO1 upon stimulation of the Nrf2-ARE pathway. These observations have important implications for the use of autologous MSCs as a potential new therapy to treat COPD.

To the best of our knowledge, this is the first study in humans that investigates whether MSCs from COPD patients are comparable to those from healthy controls. Our observation that underlying COPD does not appear to affect MSC function is in line with previous observations made in chronic systemic diseases [18-21]. In contrast, altered MSC function was observed in MSCs from patients with disorders of the central nervous system [22, 23], and altered MSCs growth and differentiation has been observed in haematologic diseases [24, 25]. Possibly, these differences might relate to the affected organ of the underlying disease, which is especially conceivable for diseases originating from the bone marrow.

MSCs secrete a spectrum of soluble factors, including cytokines, chemokines and growth factors $[2,3]$, and it was shown that this secretion differs depending on, for instance, site of origin of MSCs or systemic conditions such as hypoxaemia $[26,27]$. Likewise, a pro-inflammatory environment can "activate" MSCs, 

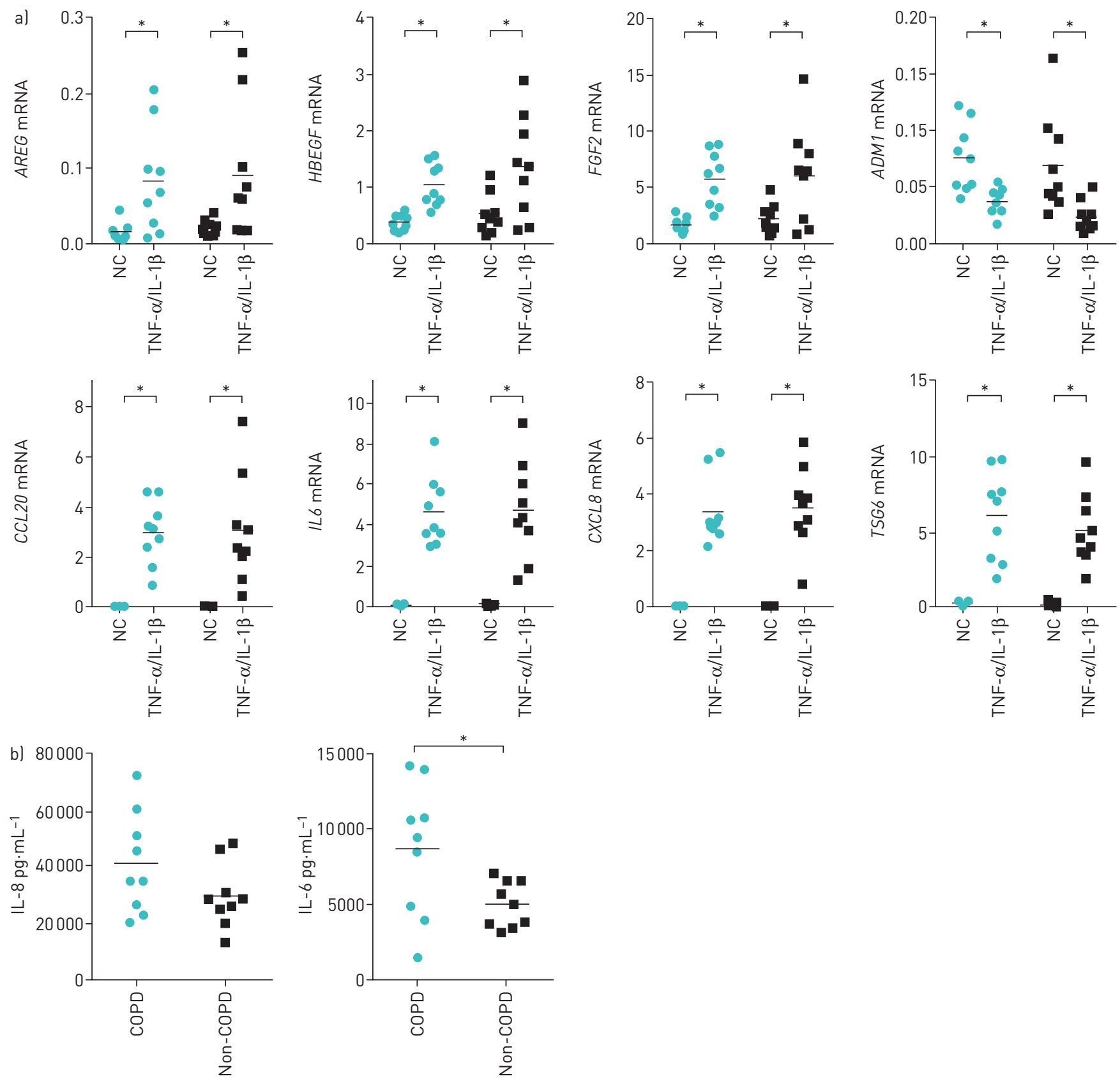

FIGURE 3 Induction of immune mediators and growth factors upon stimulation with pro-inflammatory cytokines. Mesenchymal stromal cells (MSCs) were stimulated with tumour necrosis factor (TNF)- $\alpha$ and interleukin (IL)-1 $\beta$ (20 $\mathrm{ng} \cdot \mathrm{mL}^{-1}$ each) or plain culture medium as a negative control (NC) during $6 \mathrm{~h}$. a) mRNA expression of immune mediators and growth factors, normalised for housekeeping genes, shown for chronic obstructive pulmonary disease (COPD) (circles) and non-COPD donors (squares). AREG: amphiregulin; HBEGF: heparin-binding epidermal growth factor-like growth factor; FGF2: fibroblast growth factor 2; ADM1: adrenomedullin; CCL20: chemokine (C-C motif) ligand 20; IL6: IL-6; CXCL8: chemokine (C-X-C motif) ligand 8; TSG6: TNF-stimulated gene 6. Individual data are shown in graphs, horizontal bars represent mean. *: $\mathrm{p}<0.05$, $\mathrm{n}=9$ per group. b) IL- 6 and IL-8 protein secretion by MSCs from COPD and non-COPD donors, assessed $24 \mathrm{~h}$ after addition of TNF- $\alpha$ and IL-1 3 . Individual data points are shown; horizontal bars represent mean. *: $p<0.05, n=9$ per group.

as pro-inflammatory cytokines were shown to induce expression of immune mediators and trophic factors in MSCs (reviewed in [16]). We used the pro-inflammatory cytokines TNF- $\alpha$ and IL-1 $\beta$ that are implicated in COPD pathogenesis $[28,29]$, and likewise observed induction of immune mediators and growth factors, which was unaffected by COPD status. Along with the observation that MSC-CM-induced EGFR ligand expression in airway epithelial cells was comparable between groups, these data provide evidence that MSCs from COPD patients respond in a similar fashion to inflammation. This suggests that their ability to modify inflammation and induce repair upon recruitment to inflammatory sites, such as the lung in COPD, is preserved. 

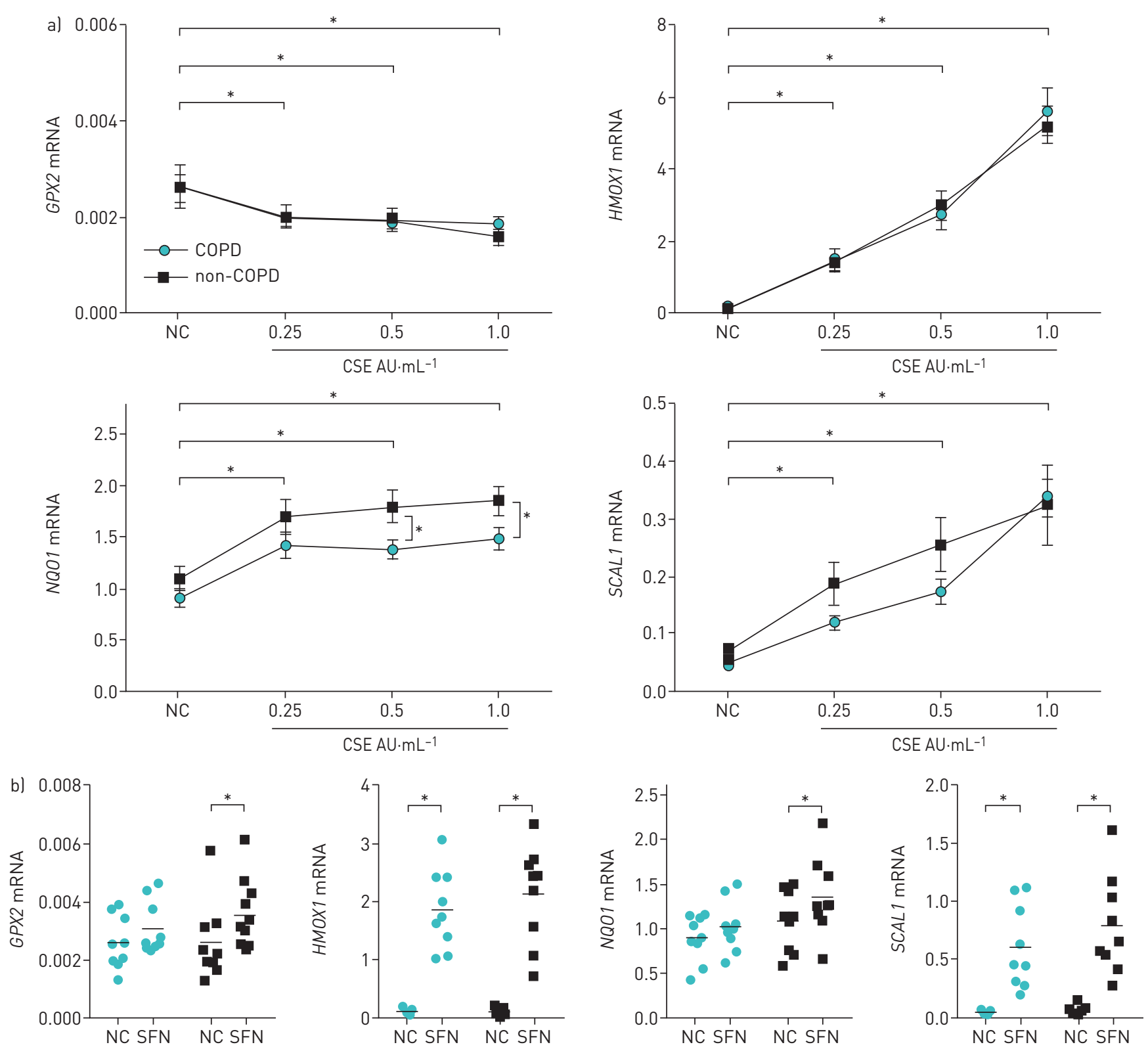

FIGURE 4 Induction of the nuclear factor (erythroid derived 2)-like 2 antioxidant response element (Nrf2-ARE) target genes in mesenchymal stromal cells (MSCs) from chronic obstructive pulmonary disease (COPD) and non-COPD donors. Gene expression of glutathione peroxidase 2 (GPX2), haem oxygenase 1 (HMOX1), NAD(P)H:quinone oxidoreductase 1 (NQ01) and smoke and cancer-associated long noncoding RNA-1 (SCAL1) was assessed in MSCs from COPD and non-COPD donors $6 \mathrm{~h}$ after addition of cigarette smoke extract (CSE) or sulforaphane (SFN), which are both inducers of the Nrf2-ARE pathway, or plain culture medium as a negative control (NC). a) Normalised mRNA expression in response to increasing concentrations of CSE. Data are presented as mean \pm SEM. ${ }^{*}: p<0.05, n=9$ per group (except CSE $1.0 \mathrm{AU} \cdot \mathrm{mL}^{-1}$ : $\mathrm{n}=7$ in $\mathrm{COPD}$ group). b) Normalised mRNA expression in response to $25 \mu \mathrm{M}$ SFN, compared with housekeeping genes. Individual data points are shown (circles: COPD; squares: non-COPD); horizontal bars represent mean. *: $p<0.05, n=9$ per group.

MSCs from COPD and non-COPD donors were phenotypically similar, and all had trilineage differentiation potential, confirming their status as progenitor cells. We found significantly higher adipocyte differentiation in MSCs from COPD patients compared with their healthy counterparts and a trend towards lower osteogenic differentiation. Our data might have even underestimated the osteogenic differentiation potential of MSCs from the non-COPD group, due to loss of the differentiated monolayer due to strong contractility of differentiated cells at the wells periphery, in a substantial subgroup of COPD and non-COPD subjects.

Similar to MSCs from COPD patients, increased adipocyte differentiation has been observed in aged MSCs and this appears to occur at the expense of osteoblast differentiation [30-32]. A definite statement about this so-called "adipogenic switch" in aged MSCs has not yet been made due to inconsistencies between studies [33]. However, if cellular ageing affects the differentiation potential of MSCs, then based on the 

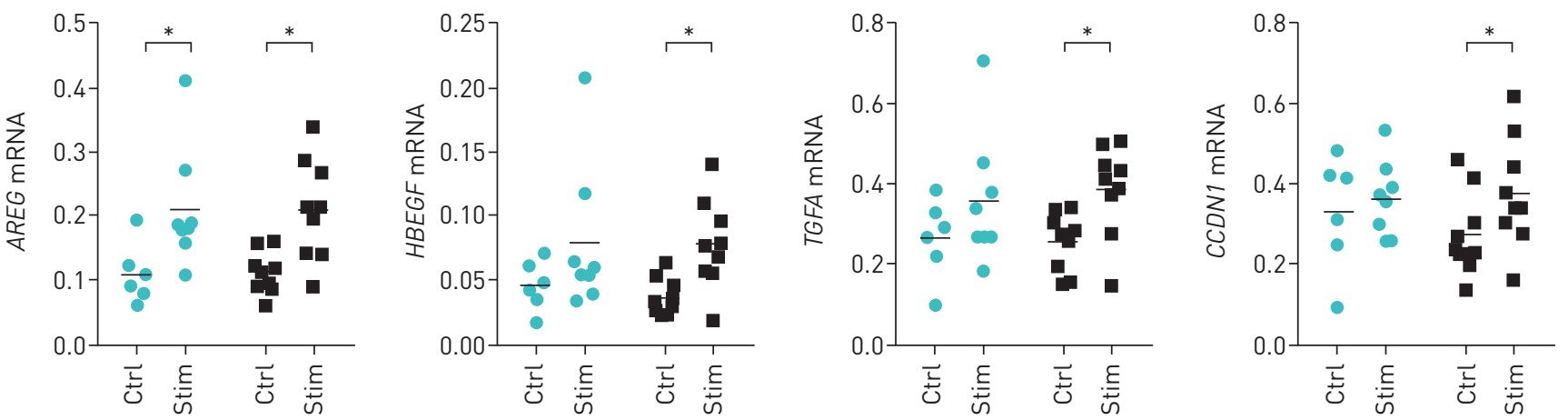

FIGURE 5 Paracrine effects of mesenchymal stromal cells (MSCs) on $\mathrm{H} 292$ airway epithelial cells. $\mathrm{NCl}-\mathrm{H} 292$ airway epithelial cells were incubated with conditioned medium obtained from MSCs (MSC-CM). mRNA expression of epidermal growth factor receptor ligands after $9 \mathrm{~h}$ incubation with conditioned medium from MSCs cultured in serum-free low-glucose Dulbecco's modified Eagle medium (LG-DMEM) (Ctrl, from MSC-CM ${ }^{\mathrm{CTRL}}$ ) or in

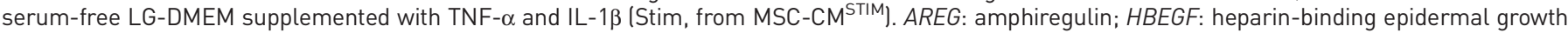
factor-like growth factor; TGFA: transforming growth factor- $\alpha$; CCDN1: cyclin D1. Normalised values compared with housekeeping genes are shown. Individual data points are shown (circles: chronic obstructive pulmonary disease (COPD) donor-derived MSC-CM; squares: non-COPD donor-derived MSC-CM); horizontal bars represent mean. *: $p<0.05, n=9$ per group. For all genes investigated, mRNA expression was lower in cells cultured in plain culture medium (LG-DMEM without additional stimulation) compared with mRNA expression in cells cultured in MSC-CM (data not shown).

current data we propose that increased cellular ageing may underlie the "adipogenic switch" we observed in MSCs from COPD patients. Interestingly, accelerated cellular ageing has been thought to underlie COPD [34]. Since oxidative stress contributes to cellular ageing [35] as well as to COPD pathogenesis [17], we speculate that oxidative stress might explain the shared features of altered differentiation between MSCs from COPD patients and aged MSCs. This hypothesis is supported by observations in aged mice MSCs that display decreased antioxidant power despite increases in proteins involved in antioxidant defence [36], which resembles observations made in COPD patients who have decreased antioxidant capacity [37], despite increased Nrf2 expression [38]. Concordantly, we observed lower expression of NQO1 and SCAL1 in MSCs from COPD patients, suggesting a lower antioxidant response upon induction of the Nrf2-ARE pathway. We found no differences in HMOX1 and GPX2 expression between groups, potentially explained by the role of other signalling pathways (c-Met and p63, respectively) $[39,40]$ in induction of these genes, whereas NQO1 and SCAL1 are thought to be more selective representatives of the Nrf2-ARE pathway [41, 42]. Whether decreased mRNA expression of Nrf2-ARE target genes also results in decreased antioxidant activity needs further elucidation before making more definite statements on this issue.

We have used MSCs from patients with severe to very severe COPD and compared these with MSCs from non-COPD controls. MSCs from the control group were obtained from donors from whom sufficient MSCs were expanded during initial cultures, thus allowing storage of excess MSCs. We cannot rule out that this created a selection bias in the control group, in favour of better MSC proliferation and potentially even function; however, such differences were not observed. We successfully prioritised on age-matching of both groups, but were unable to correct for smoking history, as all COPD patients were ex-smokers versus a majority of non- or never-smokers in the control group. Whereas our data are unlikely to be affected by acute effects of cigarette smoke on BM-MSCs (all COPD donors had quit smoking for at least 6 months before enrolment), we cannot exclude that we assessed effects on MSCs due to chronic cigarette smoke exposure in the past. Effects of chronic cigarette smoke exposure on MSCs has only been investigated to a limited extend in animal models and to the best of our knowledge this has not yet been investigated in human MSCs. Our study was not designed to address this question and therefore we cannot formally draw conclusions in this respect. However, taking into account the differences between the COPD and non-COPD group regarding smoking history, our data do not confirm previous results showing, for instance, detrimental effects of nicotine on proliferation and migration of MSCs [43].

Since Le BLANC et al.'s [44] report in 2004 describing remission of severe graft versus host disease after MSC infusion, much attention has been paid to the clinical application of MSCs in a variety of diseases, including lung diseases. In addition to promising effects of MSCs on inflammation and regeneration, the interest in MSC-based cell therapy is attributable to the relatively easy method to obtain and expand MSCs, and to their low-immunogenic status. These properties make MSCs interesting candidates for (commercial) cell therapy programmes. However, although allogeneic MSCs did not appear to induce immune responses in immunocompromised recipients, their application in immunocompetent recipients might not be ideal, as allogeneic MSCs were shown to elicit alloimmune responses in immunocompetent recipients [45], which moreover appeared to have detrimental effects on MSC function [46]. This underlines the importance of evaluating the treatment potential of autologous MCSs. The data provided by the current study support the 
implementation of autologous MSCs as a cell-based therapy for patients with chronic lung disease in future clinical trials.

In conclusion, we observed that the (immuno)phenotype and function of MSCs from patients with COPD is preserved, compared with their healthy counterparts. This finding is important in light of the potential clinical use of autologous MSCs to treat COPD patients. However, we did observe differences in differentiation potential and in activation of the Nrf2-ARE pathway that might link systemic manifestations of COPD to increased cellular ageing of MSCs.

\section{Acknowledgements}

The authors thank Renate Verhoosel and Jasmijn Schrumpf for assisting in cell culture work and performing part of the differentiation assays, Annemarie van Schadewijk for performing the PCR analysis, Bram van der Linden for performing the ELISAs (all from the Dept of Pulmonology, Leiden University Medical Center, Leiden, The Netherlands), the Center for Stem Cell Therapy for providing initial MSC cultures and collecting the FACS data and proliferation curves (Leiden University Medical Center, Leiden, The Netherlands), and Ron Wolterbeek for assisting in the statistical analysis (Dept of Medical Statistics, Leiden University Medical Center, Leiden, The Netherlands).

\section{References}

1 Dominici M, Le Blanc K, Mueller I, et al. Minimal criteria for defining multipotent mesenchymal stromal cells. The International Society for Cellular Therapy position statement. Cytotherapy 2006; 8: 315-317.

2 Keating A. Mesenchymal stromal cells: new directions. Cell Stem Cell 2012; 10: 709-716.

3 Nauta AJ, Fibbe WE. Immunomodulatory properties of mesenchymal stromal cells. Blood 2007; 110: 3499-3506.

4 Brusselle GG, Joos GF, Bracke KR. New insights into the immunology of chronic obstructive pulmonary disease. Lancet 2011; 378: 1015-1026.

5 Weiss DJ, Casaburi R, Flannery R, et al. A placebo-controlled, randomized trial of mesenchymal stem cells in COPD. Chest 2013; 143: 1590-1598.

6 Stolk J, Broekman W, Mauad T, et al. A phase I study for intravenous autologous mesenchymal stromal cell administration to patients with severe emphysema. QJM 2016; 109: 331-336.

7 Barnes PJ, Celli BR. Systemic manifestations and comorbidities of COPD. Eur Respir J 2009; 33: 1165-1185.

8 Jahn A., Rio C, Gigirey O, et al. Bone marrow-derived MSCs from patients with COPD have abnormal functional capacity. Eur Respir J 2013; 42: Suppl. 57, 22s.

9 Rankin SM. Impact of bone marrow on respiratory disease. Curr Opin Pharmacol 2008; 8: 236-241.

10 Duijvestein M, Vos AC, Roelofs $\mathrm{H}$, et al. Autologous bone marrow-derived mesenchymal stromal cell treatment for refractory luminal Crohn's disease: results of a phase I study. Gut 2010; 59: 1662-1669.

11 Ma Q. Role of Nrf2 in oxidative stress and toxicity. Annu Rev Pharmacol Toxicol 2013; 53: 401-426.

12 Sekine T, Hirata T, Mine T, et al. Activation of transcription factors in human bronchial epithelial cells exposed to aqueous extracts of mainstream cigarette smoke in vitro. Toxicol Mech Methods 2016; 26: 22-31.

13 Zhang Y, Talalay P, Cho CG, et al. A major inducer of anticarcinogenic protective enzymes from broccoli: isolation and elucidation of structure. Proc Natl Acad Sci U S A 1992; 89: 2399-2403.

14 Vandesompele J, De Preter K, Pattyn F, et al. Accurate normalization of real-time quantitative RT-PCR data by geometric averaging of multiple internal control genes. Genome Biol 2002; 3: RESEARCH0034.

15 Broekman W, Amatngalim GD, Mooij-Eijk Y, et al. TNF- $\alpha$ and IL-1 $\beta$-activated human mesenchymal stromal cells increase airway epithelial wound healing in vitro via activation of the epidermal growth factor receptor. Respir Res 2016; 17: 1-12.

16 Ma S, Xie N, Li W, et al. Immunobiology of mesenchymal stem cells. Cell Death Differ 2014; 21: 216-225.

17 MacNee W. Pulmonary and systemic oxidant/antioxidant imbalance in chronic obstructive pulmonary disease. Proc Am Thorac Soc 2005; 2: 50-60.

18 Reinders ME, Roemeling-van Rhijn M, Khairoun M, et al. Bone marrow-derived mesenchymal stromal cells from patients with end-stage renal disease are suitable for autologous therapy. Cytotherapy 2013; 15: 663-672.

19 Larghero J, Farge D, Braccini A, et al. Phenotypical and functional characteristics of in vitro expanded bone marrow mesenchymal stem cells from patients with systemic sclerosis. Ann Rheum Dis 2008; 67: 443-449.

20 Bocelli-Tyndall C, Bracci L, Spagnoli G, et al. Bone marrow mesenchymal stromal cells (BM-MSCs) from healthy donors and auto-immune disease patients reduce the proliferation of autologous- and allogeneic-stimulated lymphocytes in vitro. Rheumatology 2007; 46: 403-408.

21 Bernardo ME, Avanzini MA, Ciccocioppo R, et al. Phenotypical/functional characterization of in vitro-expanded mesenchymal stromal cells from patients with Crohn's disease. Cytotherapy 2009; 11: 825-836.

22 Cho GW, Noh MY, Kim HY, et al. Bone marrow-derived stromal cells from amyotrophic lateral sclerosis patients have diminished stem cell capacity. Stem Cells Dev 2009; 19: 1035-1042.

23 Mazzanti B, Aldinucci A, Biagioli T, et al. Differences in mesenchymal stem cell cytokine profiles between MS patients and healthy donors: implication for assessment of disease activity and treatment. J Neuroimmunol 2008; 199: $142-150$.

24 Arnulf B, Lecourt S, Soulier J, et al. Phenotypic and functional characterization of bone marrow mesenchymal stem cells derived from patients with multiple myeloma. Leukemia 2007; 21: 158-163.

25 Chao YH, Peng CT, Harn HJ, et al. Poor potential of proliferation and differentiation in bone marrow mesenchymal stem cells derived from children with severe aplastic anemia. Ann Hematol 2010; 89: 715-723.

26 Chen L, Xu Y, Zhao J, et al. Conditioned medium from hypoxic bone marrow-derived mesenchymal stem cells enhances wound healing in mice. PLoS One 2014; 9: e96161.

27 Hsiao ST, Asgari A, Lokmic Z, et al. Comparative analysis of paracrine factor expression in human adult mesenchymal stem cells derived from bone marrow, adipose, and dermal tissue. Stem Cells Dev 2011; 21: 2189-2203.

28 Lappalainen U, Whitsett JA, Wert SE, et al. Interleukin-1beta causes pulmonary inflammation, emphysema, and airway remodeling in the adult murine lung. Am J Respir Cell Mol Biol 2005; 32: 311-318. 

factor-alpha and interleukin-1 beta receptor-deficient mice. Lab Invest 2002; 82: 79-85. compromises differentiation and survival of mesenchymal stromal cells via the oxidative stress pathway. Cell $D$ eath Dis 2013; 4: e970.

31 Kim M, Kim C, Choi YS, et al. Age-related alterations in mesenchymal stem cells related to shift in differentiation from osteogenic to adipogenic potential: implication to age-associated bone diseases and defects. Mech Ageing Dev 2012; 133: 215-225.

32 Moerman EJ, Teng K, Lipschitz DA, et al. Aging activates adipogenic and suppresses osteogenic programs in mesenchymal marrow stroma/stem cells: the role of PPAR- $\gamma 2$ transcription factor and TGF- $\beta$ /BMP signaling pathways. Aging Cell 2004; 3: 379-389.

33 Sethe S, Scutt A, Stolzing A. Aging of mesenchymal stem cells. Age Res Rev 2006; 5: 91-116.

34 Ito K, Barnes PJ. COPD as a disease of accelerated lung aging. Chest 2009; 135: 173-180.

35 Finkel T, Holbrook NJ. Oxidants, oxidative stress and the biology of ageing. Nature 2000; 408: $239-247$.

36 Kasper G, Mao L, Geissler S, et al. Insights into mesenchymal stem cell aging: involvement of antioxidant defense and actin cytoskeleton. Stem Cells 2009; 27: 1288-1297.

37 Rahman I, Swarska E, Henry M, et al. Is there any relationship between plasma antioxidant capacity and lung function in smokers and in patients with chronic obstructive pulmonary disease? Thorax 2000; 55: $189-193$.

38 Pierrou S, Broberg P, O’Donnell RA, et al. Expression of genes involved in oxidative stress responses in airway epithelial cells of smokers with chronic obstructive pulmonary disease. Am J Respir Crit Care Med 2007; 175: 577-586.

39 Balan M, Teran EM, Waaga-Gasser AM, et al. Novel roles of c-Met in the survival of renal cancer cells through the regulation of HO-1 and PD-L1 expression. J Biol Chem 2015; 290: 8110-8120.

40 Yan W, Chen X. GPX2, a direct target of p63, inhibits oxidative stress-induced apoptosis in a p53-dependent manner. J Biol Chem 2006; 281: 7856-7862.

41 Korashy HM, El-Kadi AO. Transcriptional regulation of the NAD(P)H:quinone oxidoreductase 1 and glutathione $S$-transferase YA genes by mercury, lead, and copper. Drug Metab Dispos 2006; 34: 152-165.

42 Thai $\mathrm{P}$, Statt S, Chen $\mathrm{CH}$, et al. Characterization of a novel long noncoding RNA, SCAL1, induced by cigarette smoke and elevated in lung cancer cell lines. Am J Respir Cell Mol Biol 2013; 49: 204-211.

$43 \mathrm{Ng}$ TK, Carballosa CM, Pelaez D, et al. Nicotine alters microRNA expression and hinders human adult stem cell regenerative potential. Stem Cells Dev 2012; 22: 781-790.

44 Le Blanc K, Rasmusson I, Sundberg B, et al. Treatment of severe acute graft-versus-host disease with third party haploidentical mesenchymal stem cells. Lancet 2004; 363: 1439-1441.

45 Hare JM, Fishman JE, Gerstenblith G, et al. Comparison of allogeneic vs autologous bone marrow-derived mesenchymal stem cells delivered by transendocardial injection in patients with ischemic cardiomyopathy: the POSEIDON randomized trial. JAMA 2012; 308: 2369-2379.

46 Huang XP, Sun Z, Miyagi Y, et al. Differentiation of allogeneic mesenchymal stem cells induces immunogenicity and limits their long-term benefits for myocardial repair. Circulation 2010; 122: 2419-2429. 